\title{
Functional and radiological outcome after delayed fixation of femoral neck fractures in pediatric patients
}

\author{
Manish Kumar Varshney • Ashok Kumar • \\ Shah Alam Khan $\cdot$ Shishir Rastogi
}

Received: 19 May 2009/Accepted: 23 October 2009/Published online: 20 November 2009

(C) Springer-Verlag 2009

\begin{abstract}
Background Complications that develop after femoral neck fracture in children-especially osteonecrosis-have been retrospectively attributed to inadvertent delayed fixation and fracture type. Prospective evaluation of results after delayed fixation of femoral neck fractures in children beyond the first $24 \mathrm{~h}$ is not reported in the literature and requires evaluation to increase our understanding of the procedure and improve fixation methods. Also, the role of capsular decompression in initial management needs to be elucidated.

Materials and methods Radiological and functional evaluation was done for delayed fixation $(>24 \mathrm{~h})$ of displaced fractures in the femoral neck in 21 children (21 hips) treated over 11 years. Mean patient age was 11.8 (median 12, range 5-15) years. Extraphyseal fixation was done using partially threaded cannulated cancellous screws after closed or open reduction. Patients were allowed full weight bearing after 12-18 weeks. Results were assessed on the
\end{abstract}

A. Kumar $\cdot$ S. A. Khan $\cdot$ S. Rastogi

Department of Orthopaedics,

All India Institute of Medical Sciences,

Ansari Nagar, Delhi 110029, India

M. K. Varshney

Department of Orthopaedics,

Lady Harding Medical College

and associated hospitals,

New Delhi 110001, India

M. K. Varshney ( $\bowtie)$

Rm. No. 524, Masjid Moth Resident Doctors' Hostel,

Behind South-Extn-II, All India Institute of Medical Sciences,

New Delhi 110049, India

e-mail: drmkvarshney@ rediffmail.com;

drmkvarshney@gmail.com basis of modified Ratliff criteria. Patients were followed for a mean of 81 (range 66-129) months.

Results All fractures united at a mean duration of 12 (range 10.6-14) weeks. Three (14.3\%) patients had osteonecrosis of the hip, which was significantly related to poor outcome $(r=0.495 ; P=0.022)$. There was a significant correlation $(r=0.52)$ between development of osteonecrosis and delayed fracture fixation of $>10$ days $(P=0.016)$ and open reduction $(P=0.016)$.

Conclusions Outcome following temporal delay in fracture fixation of the femoral neck is primarily affected by osteonecrosis of the femoral head, whereas restriction of movements, shortening, and premature physeal closure has no significant influence. Osteonecrosis is primarily linked to delay and open reduction, whereas fracture type, age, and sex seem insignificant factors. Capsular decompression does not seem to affect the outcome in delayed presentations and may hinder definitive treatment.

Keywords Pediatric - Fracture - Femoral neck · Delayed fixation $\cdot$ Hip decompression

\section{Introduction}

Femoral neck fractures in children are uncommon, accounting for $<1 \%$ of all fractures in pediatric patients [1-3]. The treatment of choice for displaced fractures is emergent closed or open reduction and internal fixation as early as possible [1, 3-9]. Inadvertent delay in fixation may occur and are not uncommon in developing countries due to a variety of reasons. These fractures are known to have a high complication rate, including osteonecrosis of the femoral head (the most common and disabling complication), chondrolysis, nonunion, premature physeal closure, 
coxa vara, and limb-length discrepancies [2, 7, 10, 11]. Primarily, complications have been linked to delayed treatment [6-8, 10-12], fracture type and patient age [2], inadequate reduction [6], and fixation failure [9]. Causes of complications have been established by retrospective evaluation, with only a few studies documenting the development of complications after temporal delay in fixation. The role of capsular decompression also has not been reasonably established considering the fact that primary injury to vascularity occurs at the time of initial injury [13, 14]. We studied 21 cases of displaced femoral neck fractures presenting to us over a period of 11 years in a retrospective-prospective manner to determine radiological and functional outcomes of delayed fixation, factors influencing outcomes, and whether capsular decompression has a role in the initial management of these fractures.

\section{Materials and methods}

This study was conducted at our institute to evaluate the results of surgery for displaced fracture of the femoral neck in children receiving treatment beyond the commonly recommended period of $24 \mathrm{~h}$. The study was authorized by the local ethical committee and was performed in accordance with the Ethical Standards of the 1964 Declaration of Helsinki, as revised in 2000. On analyzing data from May 1997 to May 2008, 21 cases in the pediatric age group ( $\leq 16$ years) presenting after $24 \mathrm{~h}$ of injury were found (Table 1). The cases were selected sequentially from the available records. Written informed consent was obtained from patients' parents before inclusion into the study. Seventeen patients received quack treatment locally, three others were referred late by a local physician (stabilization for associated head and abdominal injury), and one patient presented late due to initial negligence by family members. Patients presenting and managed within $24 \mathrm{~h}$ of injury, having fracture dislocation of hip or associated fracture of the femoral shaft, pathological fracture, and patients with slipped capital femoral epiphysis were excluded from the study. Clinical evaluation regarding mode of injury, initial treatment received, and medicosurgical comorbidity was done. There were seven girls $(33.33 \%)$ and 14 boys $(66.67 \%)$, with an average age of 11.8 (median 12; range 5-15) years. Twelve children (57.14\%) sustained the injury in road traffic accidents (three pedestrians and nine passengers). Nine children $(42.85 \%)$ fell from a height. Fourteen patients $(66.67 \%)$ had type 2 (Figs. 1a-c, 2a, b)
Table 1 Patients with treatment delay of less than 10 days and more than 10 days

\begin{tabular}{|c|c|c|c|c|c|c|c|}
\hline Patient & Age & Sex & $\begin{array}{l}\text { Delay } \\
\text { (days) }\end{array}$ & $\begin{array}{l}\text { Type of } \\
\text { fracture }\end{array}$ & Reduction & Complication (s) & $\begin{array}{l}\text { Union } \\
\text { (weeks) }\end{array}$ \\
\hline \multicolumn{8}{|c|}{ Less than 10 days } \\
\hline$S$ & 14 & M & 2 & 2 & $\mathrm{C}$ & - & 10.9 \\
\hline $\mathrm{K}$ & 13 & $\mathrm{~F}$ & 4 & 2 & $\mathrm{C}$ & - & 10.9 \\
\hline $\mathrm{T}$ & 14 & $\mathrm{~F}$ & 5 & 2 & $\mathrm{C}$ & - & 10.6 \\
\hline I & 12 & M & 6 & 3 & $\mathrm{C}$ & $\begin{array}{l}\text { Pre-mat physeal } \\
\text { closure }+ \text { shortening }\end{array}$ & 11 \\
\hline $\mathrm{J}$ & 13 & M & 2 & 2 & $\mathrm{C}$ & - & 13.4 \\
\hline $\mathrm{L}$ & 14 & M & 4 & 3 & $\mathrm{C}$ & - & 10.7 \\
\hline $\mathrm{Y}$ & 5 & $\mathrm{~F}$ & 5 & 2 & $\mathrm{C}$ & - & 14 \\
\hline G & 12 & M & 6 & 2 & $\mathrm{C}$ & - & 11 \\
\hline $\mathrm{R}$ & 9.5 & M & 2 & 2 & $\mathrm{C}$ & - & 11 \\
\hline $\mathrm{U}$ & 8.5 & M & 7 & 2 & $\mathrm{C}$ & - & 13 \\
\hline $\mathrm{L}$ & 14 & $\mathrm{~F}$ & 3 & 2 & $\mathrm{C}$ & - & 11 \\
\hline Q & 15 & M & 9 & 2 & $\mathrm{C}$ & - & 11 \\
\hline $\mathrm{N}$ & 12 & M & 5 & 3 & $\mathrm{C}$ & - & 12 \\
\hline \multicolumn{8}{|c|}{ More than 10 days } \\
\hline M & 11 & M & 11 & 3 & $\mathrm{O}$ & - & 11.1 \\
\hline $\mathrm{L}$ & 8 & M & 14 & 2 & $\mathrm{O}$ & Osteonecrosis & 12.6 \\
\hline $\mathrm{K}$ & 11 & $\mathrm{~F}$ & 12 & 2 & $\mathrm{O}$ & - & 12 \\
\hline $\mathrm{D}$ & 15 & M & 22 & 2 & $\mathrm{O}$ & Osteonecrosis + shortening & 13.3 \\
\hline $\mathrm{S}$ & 12 & $\mathrm{~F}$ & 13 & 3 & $\mathrm{O}$ & - & 10.9 \\
\hline I & 11 & M & 11 & 3 & $\mathrm{O}$ & Osteonecrosis & 12.2 \\
\hline A & 13 & M & 15 & 2 & $\mathrm{O}$ & - & 10.6 \\
\hline $\mathrm{J}$ & 12 & $\mathrm{~F}$ & 12 & 3 & $\mathrm{O}$ & - & 11.3 \\
\hline
\end{tabular}


and seven $(33.33 \%)$ type 3 fractures according to DelbetColonna classification. The average delay in presentation was 8.1 (range 2-22) days. The average delay to treatment from presentation was $17.1 \mathrm{~h}$ (range $11-31 \mathrm{~h}$ ). In thirteen children $(61.9 \%)$, percutaneous screws were inserted following successful closed reduction. Eight children $(38.09 \%)$ required open reduction (with invariable decompression of capsule) following failed closed reduction. These patients presented after 10 days of injury. The patient's limb was immobilized in a Thomas splint with fixed skin traction until definitive treatment. Anteroposterior radiographs of the pelvis with both hips and cross-table lateral radiographs of the affected hip were studied in all patients. Closed reduction (by longitudinal traction, abduction, and internal rotation) was attempted in all cases on a fracture table, with simultaneous preparation for open reduction in the event the former procedure failed. As a protocol in our institute, miniarthrotomy or decompression was not done in fractures treated by closed reduction presenting after $24 \mathrm{~h}$. Open reduction was done using a anterolateral approach to the hip in cases that failed to reduce by closed maneuvers. An extraphyseal fixation was performed with two (three patients) or three (17 patients) 4$\mathrm{mm}$ partially threaded (16-mm thread length) cannulated cancellous screws. The screw length was decided intraoperatively under image intensifier guidance, and screws were placed in inverted triangle configuration. All children were given a hip spica cast in the postoperative period for 6 weeks. After cast removal, the children were kept nonweight bearing for 2 weeks (in-bed mobilization) followed subsequently by toe touch weight bearing for a couple of weeks, gradually increasing to partial weight bearing (up to $50 \%$ ) over 4-6 weeks. Full weight bearing was allowed only after 12 weeks of surgery. Children were followed up on a regular basis every 3 months for next 2 years then every year till closure of the physis. The mean follow-up was 81 (range 66-129) months. Treatment completion was defined as radiological union in two perpendicular planes. Treatment failures for the purpose of study were defined as implant failure and/or fracture displacement to an unacceptable position before union. Functional outcome was assessed using the modified Ratliff criteria [1] at capital physis closure or 5 years of follow-up, whichever was longer; dividing results into good, fair, and poor. Clinically, the patients were assessed for pain and range of motion.

Chi-square analysis was used to identify the factors affecting functional outcome. Ordinal correlation statistics (Spearman correlation) were used to find association of delay in fracture fixation and type of reduction to development of osteonecrosis. Null hypothesis that capsular decompression does not result in reduction of the risk of osteonecrosis was formulated and analyzed using ordinal correlation and chi-square analysis. Also, multinomial logistic regression analysis was used to find if there was any influence of age, sex, or fracture type to the development of osteonecrosis. Significance was set at $P<0.05$ (95\% confidence interval).

\section{Results}

All the fractures united with an average time to radiological union (Figs. 1, 2) of 12 (range 10.6-14) weeks. Osteonecrosis was seen in three cases $(14.3 \%)$ at 18,21 , and 33 months of follow-up, respectively. These three patients were treated after 10 days of injury and required open reduction. Two had pain and delayed limitation of rotation and flexion even after fracture union; one was asymptomatic.
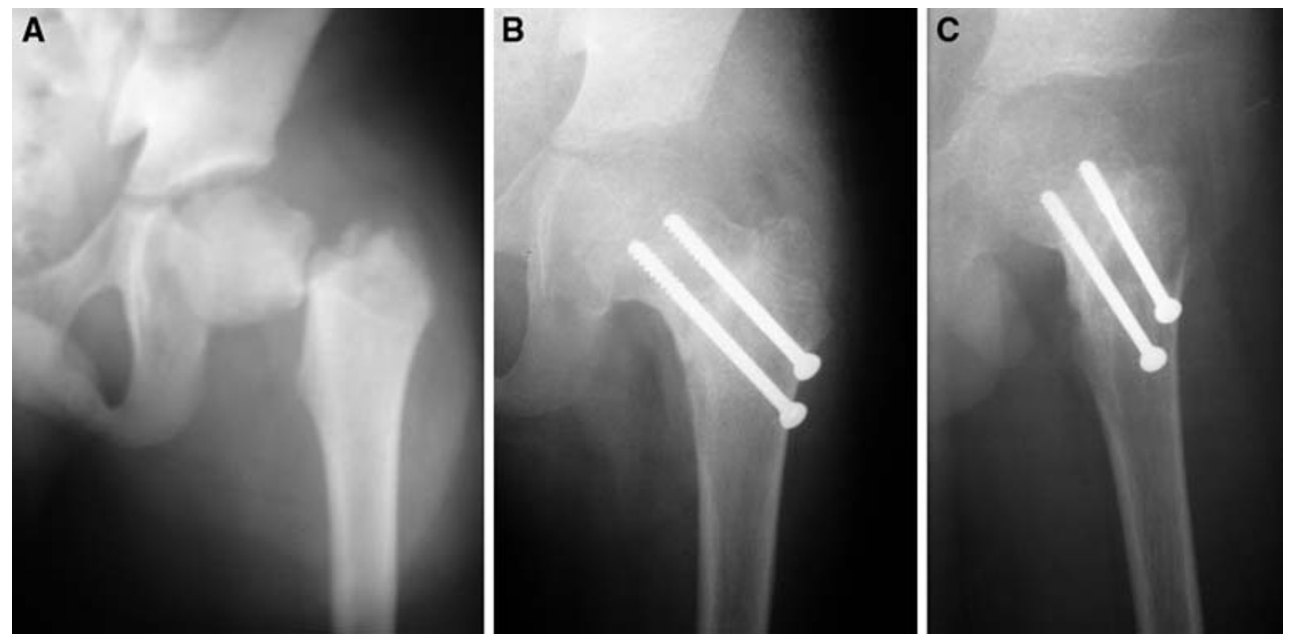

Fig. 1 a Anteroposterior radiograph (preoperative) of left hip with thigh showing type 2 fracture of the femoral neck. b Anteroposterior radiograph (postoperative) of left hip with thigh showing united femoral neck fracture with cancellous screws in situ. c Lateral radiograph (postoperative) of left hip with thigh showing united fracture of the femoral neck in the same child 

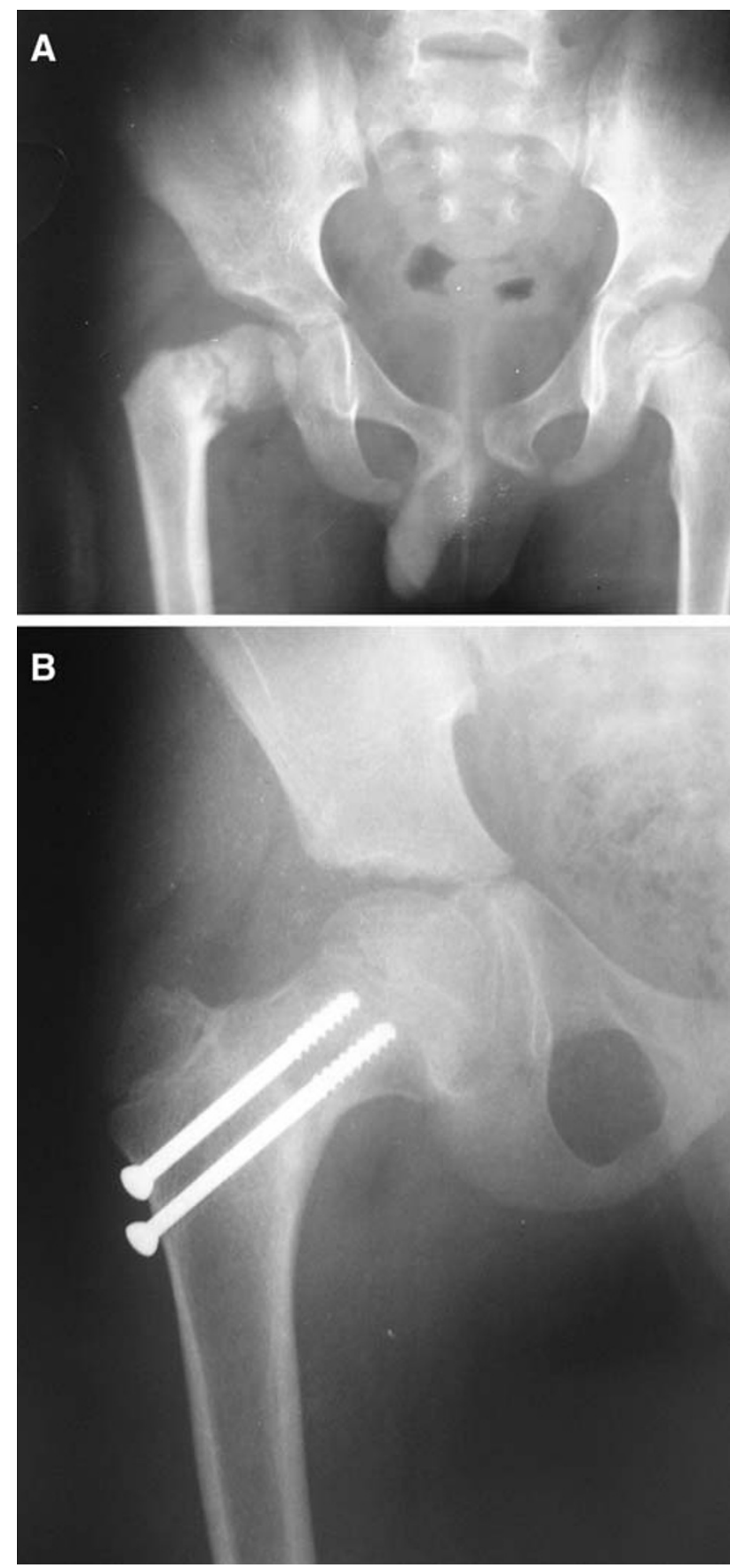

Fig. 2 a Anteroposterior radiograph (preoperative) of pelvis with both hips showing a 15-day-old type 2 fracture of right-sided femoral neck. b Anteroposterior radiograph (postoperative) of right hip with thigh showing united fracture of the femoral neck after open reduction and fixation with cancellous screws

Two patients had type 2 osteonecrosis and one had type 1 osteonecrosis according to Ratliff classification [1]. There was a significant correlation [Spearman Rho $(r)=0.52$ ] between development of osteonecrosis and fracture fixation delayed by more than 10 days $(P=0.016)$ and open reduction $(P=0.016)$. Multinomial regression analysis found no influence of age $(P=0.873)$, sex $(P=0.998)$, or type of fracture $(P=1)$ to the development of osteonecrosis. There was no correlation $(r=-0.149)$ between development of osteonecrosis in patients either younger or older than 10 years $(P=0.496)$. There was a very strong statistical correlation $(r=1.0)$ for requirement of open reduction and delay in presentation of $>10$ days $(P=0.000)$. Symptomatic osteonecrosis was treated with core decompression and fibular grafting, whereas asymptomatic lesions were followed with non-weight bearing for 3 months, which spontaneously improved radiologically.

To find the influence of capsular decompression, patients who were treated with closed reduction were analyzed. There was no correlation $(r=0.04)$ between patients who were managed with closed reduction and the development of osteonecrosis $(P=0.863)$, and the odds ratio (OR) for development of osteonecrosis in such patients was 0.786 (relative risk for osteonecrosis $=1.231)$. Thus, the null hypothesis was supported.

Eleven patients (52.4\%) had good, seven $(33.33 \%)$ fair, and three (14.3\%) poor results (two had osteonecrosis and one had premature physeal closure). There was a significant association $(r=0.495)$ between a poor result and osteonecrosis $(P=0.022$, likelihood ratio $=3)$. Shortening (average $2.1 \mathrm{~cm}$ ) of the affected limb was seen in two patients (one had premature physeal closure; the other was associated with osteonecrosis of the femoral head). One patient with shortening had a poor result, but there was no relation $(r=0.256)$ between shortening and the poor result $(P=0.241)$. Premature closure of the physis was seen in one patient and had a poor result, but no correlation could be computed. Average loss of $10^{\circ}$ of terminal extension (all painless) was noted at the last follow-up in eight patients treated with open reduction and fixation, but there was no significant correlation $(r=0.329)$ to poor result $(P=0.108)$. There was no unstable hip at follow-up. No patient had nonunion, infection, coxa vara (neck shaft angle $<120^{\circ}$ ), or chondrolysis. Eighteen patients who had good to fair results were free of pain.

\section{Discussion}

Fracture of the femoral neck is quite uncommon in children [1-4], and most orthopaedic surgeons have the opportunity to treat such fractures very few times during their career. The literature recommends early and aggressive treatment for optimum results and to avoid a high rate of complications associated with this fracture [1, 4-11]. However, delay in receiving definitive treatment is a common occurrence in the developing world. The main causes are illiteracy, the urge to receive cheap quack treatment due to poverty and lack of more scientific resources in 
far-reaching regions, and sometimes late referral by hospitals or the general physician from remote areas. In our study, all the children received femoral neck fracture from high-energy trauma. Our aim in this study was to evaluate outcome following delayed fixation of pediatric femoral neck fractures in an attempt to establish a cause-effect relationship and the factors influencing the outcome. In addition, we analyzed the effect of joint decompression on osteonecrosis development.

Overall prevalence of osteonecrosis of the femoral head in children has been reported to be around $30 \%$ based on nine series in the literature [2, 6, 10-12]. The incidence of osteonecrosis of the femoral head (14.3\%) and premature closure of the physis $(4.8 \%)$ in our study was similar to that reported by the Togrul et al. [8], which is lower than the overall incidence (30\%). Togrul et al. [8] reported osteonecrosis in nine patients $(14.5 \%)$ and premature epiphysis fusion in five (8\%) of 61 patients. Bombaci et al. [7] reported osteonecrosis in 12 patients $(54.6 \%)$ and premature physeal closure in $14(63.6 \%)$. Apparently, the results of surgery performed after the first $24 \mathrm{~h}$ are mainly affected by osteonecrosis of the femoral head. There was no significant association between shortening and poor result, whereas premature physeal closure could not be evaluated for any patient.

Development of osteonecrosis was significantly correlated independently to open reduction $(P=0.016)$ and treatment delay $>10$ days $(P=0.016)$. This correlation, although statistically significant, should be carefully evaluated, as all the three patients were treated after 10 days and with open reduction. Delay in treatment has been proposed as an important factor for the development of osteonecrosis [6-9]. We support the finding that delay in treatment is a significant factor for development of osteonecrosis however open reduction of pediatric femoral neck fractures also seems to play an important role as found in our study. Fracture type, age [2], and type of treatment [9] received can definitely have compounding influences. Two patients with type 2 fracture and one with type 3 developed osteonecrosis; however, there was no significant association $(P=1.0)$, and no correlation could be found with age. We found no increased chance of osteonecrosis with increasing age and patients $>10$ year old, as was suggested by Moon et al. [2]. Fracture displacement is an interesting hypothesis [6], but universally acceptable criteria to judge displacement need to be laid down before they can be compared to complications.

Early decompression of the joint capsule has been suggested in various studies to reduce chances for development of osteonecrosis [13, 14]; however, it's pertinence to fractures presenting late needs a case-controlled prospective study. In our study, none of the patients were treated with miniarthrotomy or needle decompression. The patients in the open reduction group were the only ones receiving hip decompression as a part of procedure. These patients had no better outcome than patients treated with closed reduction, as all three cases of osteonecrosis were treated with open reduction. We found no significant correlation between osteonecrosis and closed treatment. The relative risk for osteonecrosis development in patients not surgically decompressed is 1.231 , whereas for patients treated with open reduction (surgical equivalent to decompression), it is 0.812 . This is contrary to the proposal made by other studies $[13,14]$. Our results could well be compounded by the possibility of capsular tear following high-energy trauma and self-decompression. Also, early decompression is itself influenced by temporal bias, as these fractures are also treated on an urgent basis [2, 13, 14]. The poor outcome, as discussed here, is primarily linked with time delay in definitive treatment. Osteonecrosis due to jeopardized vascular supply seems to be a consequence of injury to the vessels at the time of injury. Treatment on an emergent basis helps restore blood supply in cases with uninjured but kinked vessels. Decompression is hence of minimal importance in delayed presentations (after $24 \mathrm{~h}$ ), as normally, bone can sustain ischemia for only 4-6 h.

In our study, 18 patients $(85.71 \%)$ had good to fair results and three $(14.3 \%)$ had poor results on the basis of the modified Ratliff criteria, primarily affected by osteonecrosis $(P=0.022)$. Terminal extension was limited (average $10^{\circ}$ ) in patients treated by open reduction and internal fixation. Range of motion was a poor predictor $(P=0.108)$ for poor outcome. Most patients took 3-6 months to regain full movement. Also, flexion and sometimes even rotation may be initially comparable in patients with osteonecrosis to those without. Similar comparable results were reported by Bombaci et al. [7] and Togrul et al. [8] in their patients treated by delayed surgical treatment. Bombaci et al. [7] evaluated the results of delayed surgical treatment $(>24 \mathrm{~h})$ in 22 patients at a mean follow-up of 73 months on the basis of modified Ratliff criteria and reported fair to good results in $20(91.0 \%)$, with poor results in two (9.1\%). Togrul et al. [8] evaluated late treatment results at a follow-up of 96 months in 61 out of 102 children with femoral neck fracture and reported good radiological results in $67.2 \%$.

Shortening does not seem to affect the functional outcome $(P=0.241)$. Shortening due to premature physeal closure or osteonecrosis can both be treatment-related or an intrinsic complication of failure to reestablish vascularity (osteonecrosis) or hypervascularity and premature physeal closure. However, the exact association for development of shortening whether primarily due to injury (and consequential osteonecrosis) or because of treatment cannot be easily documented. The issue of when osteonecrosis develops can be partially settled by magnetic resonance 
imaging or technetium-99 scintigraphic scanning before fixation in order to document either injury-related osteonecrosis [15] or treatment-related osteonecrosis. However, neither of these methods are $100 \%$ specific and, more importantly, lead to further delay in treatment. In our study, shortening developed in only one of three cases with osteonecrosis; one patient had type 2 and the other had type 3 fracture. Shortening appears to be independent fracture type or osteonecrosis.

Early results after delayed surgical treatment may not show a significant difference in comparison with early fixation [2, 6-12]. All children with a femoral neck fracture should be followed up until closure of the physis for complete documentation of end results $[2,6,10,16]$. Longterm follow-up is essential for two reasons: first, osteonecrosis in the initial stages and typically in types 2 and 3 fractures (Ratliff classification) may be asymptomatic [9-12], for which conservative management may suffice. Second, we believe that there is no definitive time period until when osteonecrosis can develop, and we had two cases with radiological identification of osteonecrosis during the second year of follow-up. Patients with osteonecrosis had typical clinical signs, which can help in early detection. Persistence of pain even after spica removal, especially in the groin, is a good warning sign, which was present in two cases in our study. These two patients were also reluctant to actively cooperate in rehabilitation and weight-bearing training. This should prompt the physician to actively search for the reason. The results show that delayed fixation of femoral neck fractures in children even outside the recommended period of $24 \mathrm{~h}$ can have an acceptable functional outcome. However, this by no means should urge physicians to wait and unnecessarily delay treatment, which should be undertaken at the earliest possible time.

Though the power of our study is low (limited number of patients), results of delayed fixation seem to be mainly affected by osteonecrosis of the femoral head, which may mostly be temporally related to the delay and method of reduction, whereas fracture type, age, and sex possibly have insignificant or secondary roles. Capsular decompression does not seem to influence the outcome in delayed presentations, and primary attention should be given to definitively treat the patient.

Conflict of interest statement No financial or any other support has been received from any organization or institution. There are no conflicting interests, and the manuscript is not under consideration elsewhere for publication.

\section{References}

1. Ratliff AHC (1962) Fractures of the neck of the femur in children. J Bone Joint Surg Br 44:528-542

2. Moon ES, Mehlman CT (2006) Risk factors for avascular necrosis after femoral neck fractures in children: 25 Cincinnati cases and meta-analysis of 360 cases. J Orthop Trauma 20:323329

3. Forster NA, Ramseier LE, Exner GU (2006) Undisplaced femoral neck fractures in children have a high risk of secondary displacement. J Pediatr Orthop B 15:131-133

4. Quick TJ, Eastwood DM (2005) Pediatric fractures and dislocations of the hip and pelvis. Clin Orthop Relat Res 432:87-96

5. Forster NA, Ramseier LE, Exner GU (2006) Undisplaced femoral fractures in children have a high risk of secondary displacement. $\mathrm{J}$ Pediatr Orthop B 15(2):131-133

6. Shrader MW, Jacofsky DJ, Stans AA, Shaughnessy WJ, Haidukewych GJ (2007) Femoral neck fractures in pediatric patients: 30 years experience at a level 1 trauma center. Clin Orthop Relat Res 454:169-173

7. Bombaci H, Centel T, Babay A, Turkmen IM (2006) Evaluation of complications of femoral neck fractures in children operated on at least 24 hours after initial trauma. Acta Orthop Traumatol Turc 40(1):6-14

8. Togrul E, Bayram H, Gulsen M, Kalaci A, Ozbarlas S (2005) Fractures of the femoral neck in children: long term follow up in 62 hip fractures. Injury 36(1):123-130

9. Hughes LO, Beaty JH (1994) Current concept review: fractures of the head and neck of the femur in children. J Bone Joint Surg Am 76(2):283-292

10. Morsy HA (2001) Complications of fracture of the neck of the femur in children. A long-term follow-up study. Injury 32(1):4551

11. Bagatur AE, Zorer G (2002) Complications associated with surgically treated hip fractures in children. J Pediatr Orthop B 11:219-228

12. Pape HC, Krettek C, Friedrich A, Pohlemann T, Simon R, Tscherne H (1999) Long-term outcome in children with fractures of the proximal femur after high-energy trauma. J Trauma 46:5864

13. Song KS, Kim YS, Sohn SW, Ogden JA (2001) Arthrotomy and open reduction of the displaced fracture of the femoral neck in children. J Pediatr Orthop B 10(3):205-210

14. Cheng JC, Tang N (1999) Decompression and stable internal fixation of femoral neck fractures in children can affect the outcome. J Pediatr Orthop 19:338-343

15. Poggi JJ, Callaghan JJ, Spritzer CE (1995) et al. (1995) Changes on magnetic resonance images after traumatic hip dislocation. Clin Orthop Rel Res 319:249-259

16. Flynn JM, Wong KL, Yeh GL, Meyer JS, Davidson RS (2002) Displaced fractures of the hip in children: Management by early operation and immobilisation in hip spica cast. J Bone Joint Surg Br 84:108-112 